\title{
Gradhiva
}

GRADHIV

Revue d'anthropologie et d'histoire des arts

$32 \mid 2021$

Livres sorciers

\section{Charlotte Marchina, Nomad's Land : éleveurs, animaux et paysages chez les peuples mongols}

Bruxelles, Zones sensibles, 2019

\section{Anthony Pecqueux}

\section{(2) OpenEdition}

\section{Journals}

Édition électronique

URL : https://journals.openedition.org/gradhiva/5794

DOI : 10.4000/gradhiva.5794

ISSN : 1760-849X

\section{Éditeur}

Musée du quai Branly Jacques Chirac

Édition imprimée

Date de publication : 24 mars 2021

Pagination : 223-224

ISBN : 978-2-35744-132-3

ISSN : 0764-8928

\section{Référence électronique}

Anthony Pecqueux, "Charlotte Marchina, Nomad's Land : éleveurs, animaux et paysages chez les peuples mongols », Gradhiva [En ligne], 32 | 2021, mis en ligne le 02 avril 2021, consulté le 25 mai 2021. URL http://journals.openedition.org/gradhiva/5794 ; DOI : https://doi.org/10.4000/gradhiva.5794

Ce document a été généré automatiquement le 25 mai 2021.

(c) musée du quai Branly 
Charlotte Marchina, Nomad's Land: éleveurs, animaux et paysages chez les peuples mongols

Bruxelles, Zones sensibles, 2019

Anthony Pecqueux

\section{RÉFÉRENCE}

Charlotte Marchina, Nomad's Land : éleveurs, animaux et paysages chez les peuples mongols. Bruxelles, Zones sensibles, 2019, $219 \mathrm{p}$. 


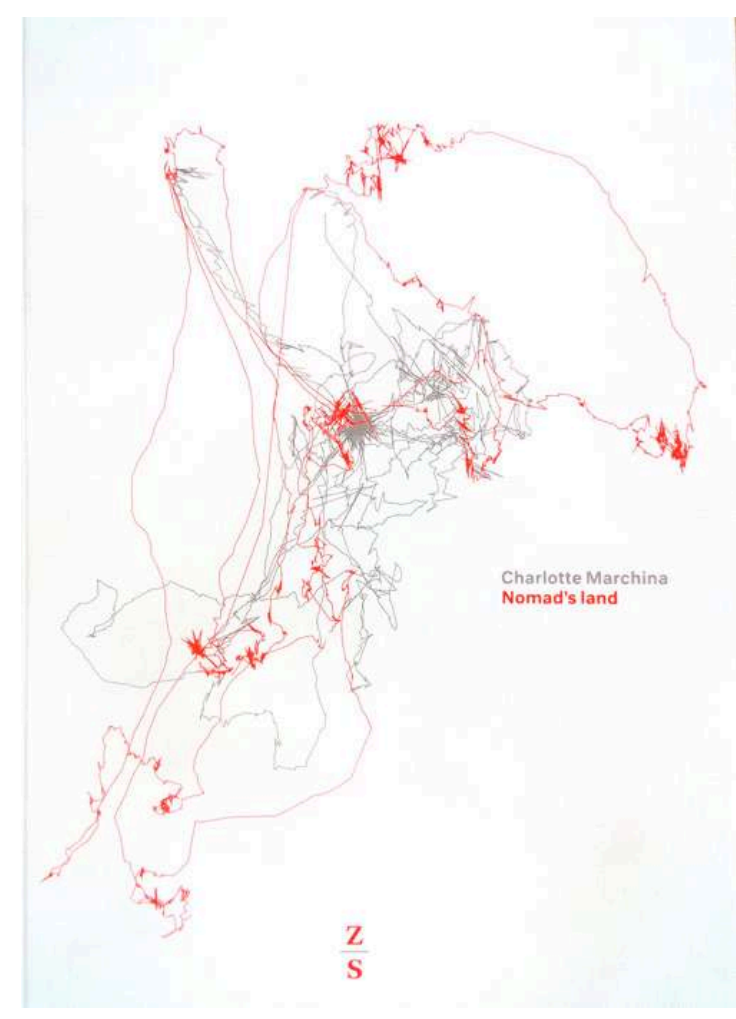

1 La première qualité de ce livre est l'objet en lui-même, que l'on doit tant à l'auteure qu'à son éditeur, Zones sensibles, qui réalise une fois de plus un beau travail, soigné sur tous les plans. À cet endroit, l'apport de Charlotte Marchina tient aux photographies très réussies qui jalonnent l'ouvrage et accompagnent notre imaginaire de lectrices et lecteurs, ainsi qu'aux nombreuses «cartes» qui documentent son travail - dont la pagination aurait cependant gagné à être mieux référencée dans le corps du texte. Lire Nomad's Land revient donc à faire très littéralement l'expérience d'une "zone sensible». En effet, Charlotte Marchina réalise l'anthropologie d'un monde crépusculaire, c'est-à-dire d'un monde dont le mode d'existence est de se présenter selon les traits d'un "quasi avoir été ", à l'état de ruine ou vestige. Si cette approche n'est pas explicitement défendue par l'auteure, elle me semble néanmoins autorisée par le livre et c'est celle que je compte adopter ici.

2 Ainsi, l'apprentie anthropologue qui voulait faire une thèse sur des nomades bouriates vivant dans des yourtes ne trouve rien de tel. Au fil des échanges, ses interlocuteurs finissent en revanche par convenir qu'ils sont des «éleveurs qui nomadisent » (p. 13) mais ils ne sont même pas mongols, tout juste "de langue et de culture mongoles". Finalement, le terrain s'est déroulé dans deux régions, auprès de Halh de Mongolie et de Bouriates de Russie. Cette ambiance crépusculaire renforcée, comme dans toute la région, par la présence fantomatique de l'URSS, se ressent encore dans le passage d'une société où la collectivisation des propriétés, infrastructures, et des modes de gestion des récoltes et des troupeaux est de mise, à une individualisation croissante de l'organisation sociale, souvent à marche forcée. Dans ce cadre, la problématique de Charlotte Marchina s'articule autour d'une triade humain-e-s/animaux/environnement qui se révèle être autant un slogan politique national (p. 203) qu'un lieu commun anthropologique. Pour elle, l'enjeu est plutôt de complexifier le récit (très moderne) d'une relation symbiotique entre les différents éléments de la triade. 
3 Ce livre s'articule autour de quatre chapitres bien délimités; le premier présente les différentes façons de nomadiser: celles des Mongols comme des Bouriates. Les premiers réalisent, en Mongolie centrale, entre deux et quatre nomadisations par an, sur de courtes distances. Charlotte Marchina montre que l'absence de propriété privée sur les espaces de pâturage autorise une certaine flexibilité, des arrangements, voire des utilisations collectives de ces espaces. Cette souplesse est rendue d'autant plus nécessaire par l'environnement et ses modifications dans le temps: des points d'eau disparaissent, des pâturages se dégradent... De manière générale, les Mongols qui nomadisent ont appris à craindre les zud, ces catastrophes d'origine climatique conduisant à une variation très importante des températures et des précipitations, dans un sens comme dans l'autre (trop ou pas assez; p. 48). Les Bouriates, de leur côté, connaissent un important mouvement de sédentarisation: ils ne font plus en effet qu'une seule nomadisation l'été, courte de surcroît et de plus en plus souvent réalisée uniquement par leurs auxiliaires russes. L'histoire est intéressante car elle montre des éleveurs aux prises avec l'institution, cherchant à faire perdurer la collectivisation héritée de la période soviétique. En effet, pour contourner l'obligation de privatisation des terres en 1995, ils ont joué du grand flou concernant à la fois les troupeaux et les infrastructures, afin de maintenir une structuration collective de leurs activités. Mais en 2011 cette obligation est devenue incontournable (p. 66). Pour autant, la surface de la parcelle allouée étant systématiquement moindre que celle nécessaire à la pâture de leurs troupeaux, la plupart des éleveurs ont mis en œuvre des stratégies d'alliance pour accroître cette surface. D'où la question inévitable : ces alliances, reposant sur la bonne entente avec les voisins et la parenté, peuvent-elles perdurer alors que la sédentarisation ne va pas sans l'apparition de conflits de voisinage (p.72) ? Voilà encore une façon d'entonner une sorte de chant du cygne en affirmant que «nomadiser, c'est "bien" » (p. 76), ou en se traitant de "paresseux» parce qu'on nomadise moins (p. 71).

4 Le deuxième chapitre est consacré à l'exploration du nutag ou nyutag (en mongol ou bouriate), qu'on peut traduire par "pays» ou home en anglais, puisque la notion s'applique aussi bien à la maison qu'au pays. Un milieu de vie, en somme, partagé entre les humain-e-s, les animaux et les entités invisibles, qui s'entremêlent à travers des processus "d'attachement et d'habituation" (p. 79) très bien décrits par Charlotte Marchina. Par exemple, elle montre comment le fait que certaines espèces d'animaux "fuguent », tels les chevaux, n'est pas un problème s'ils reviennent d'eux-mêmes au campement - c'est-à-dire s'ils montrent qu'ils sont indépendants. Cela contribue à dessiner une cartographie des espèces suivant leur degré d'autonomie. Plus généralement, se révèle ici une "dynamique d"'adaptation mutuelle" " entre territoires, éleveurs et troupeaux, à partir de l'élément partagé entre ces trois entités : les pratiques de nomadisation propres à chacune. Cette adaptation réciproque se lit y compris dans le paysage, à travers l'apparition de sentiers (suite aux piétinements des bêtes et aux passages de véhicules) qui sont autant d'«empreinte[s]»(p. 89) de ces relations.

5 Dans le troisième chapitre, Charlotte Marchina s'intéresse à l'absence d'enclos dans la steppe, qui induit précisément des partages différenciés de l'espace, suivant les espèces. $\mathrm{Y}$ interviennent en particulier les cartes des déplacements des troupeaux réalisées à partir de relevés GPS obtenus grâce à la fonction traceur. Ces cartes, évoquant les lignes d'erre que Fernand Deligny reconstitue avec son équipe pour les déplacements des 
jeunes autistes, nous donnent à voir en quelque sorte les repères pratiques des éleveurs pour évaluer l'autonomie ou l'indépendance relative des différentes espèces et ajuster en fonction leurs pratiques de surveillance : les chevaux, mais aussi les chameaux voire les bovins adultes sont considérés comme les espèces les plus autonomes (au contraire des chèvres et spécialement des moutons, gardiennés presque en permanence). Les éleveurs se servent de ces repères pratiques dans de nombreuses circonstances et selon différents enjeux, et ce d'autant plus que le cadre d'élevage est multispécifique: connaître le temps mis par chaque espèce pour atteindre un point d'eau, y envoyer d'abord les chevaux l'hiver car ils sont les seuls à pouvoir briser la glace avec leurs sabots, etc. L'absence d'enclos et des surveillances intermittentes obligent les éleveurs mongols à accomplir des tâches régulières de recherche des troupeaux égarés, ou d'individus tant humain-e-s que non-humain-e-s (p. 147). Ce pistage, réalisé à partir d'indices et traces puisés dans l'environnement, n'est un paradigme pour les sciences humaines et sociales (de Carlo Ginsburg ou Albert Piette, à plus récemment Anna L. Tsing ou Baptiste Morizot) que dans la mesure où il s'agit d'une pratique ordinaire, quotidienne. Enfin, une autre modalité de la nature sensible et sensorielle des liens (ou de leur absence) avec les troupeaux est devenue manifeste dans le passage de la yourte à la maison en dur. En effet, si le feutre de la yourte est un excellent isolant contre le froid et la chaleur, il laisse passer les sons, ce qui donne (ou donnait) une importance particulière à l'écoute dans la surveillance des troupeaux; désormais, les maisons ont tamisé ce lien sonore mais accru le rôle de la vue du fait des fenêtres. Cela signifie aussi que la nuit, les liens se sont coupés entre éleveurs et animaux.

6 Le dernier chapitre, "Animaux et territoires ", reprend, comme son titre l'indique, la problématique engagée entre espaces et espèces. S'il aborde de nombreuses pratiques, telles les hybridations expérimentées par les éleveurs mongols selon une logique de cumulation (par exemple en croisant vaches et yaks afin d'obtenir un lait à la fois plus abondant comme celui des vaches et plus riche en matières grasses comme celui des yaks), c'est pour autant essentiellement à travers les chiens que j'en rendrai compte. Leur rôle premier est de protéger le campement, ce qui conduit les éleveurs à les dresser pour être agressifs (ils vont jusqu'à limiter leur nourriture pour entretenir cet état). D'où, sur le campement, la façon de saluer du nouvel arrivant, qui dit «Retiens le(s) chien(s)! » (p. 175), pour s'assurer de l'absence d'intention belliqueuse de la part d'autrui - comme Malinowski l'avait noté pour la communication phatique : les chiens entrent ainsi paradoxalement dans le mode de communication entre humain-e-s. Plus généralement, le comportement des éleveurs vis-à-vis des chiens manque singulièrement de tendresse (ils sont maintenus dehors, sous-alimentés...) ; à nouveau, les auxiliaires russes prennent le relais en s'occupant d'eux, en leur parlant, en leur prodiguant des caresses, en nouant des relations affectives entre "espèces compagnes » (selon l'expression de Donna Haraway ${ }^{1}$ 2019). Pour les éleveurs bouriates, voilà tous les signes d'une russification croissante, non seulement sur le plan linguistique (les chiens ne comprendraient plus les ordres en bouriate) mais aussi comportemental, puisque là où chiens et auxiliaires russes pourraient y voir une forme d'attachement réciproque, les éleveurs y lisent une "perte d'autonomie des chiens » (p. 180).

7 Comprendre cela comme une forme de nationalisme anti-russe serait une interprétation pauvre; je souhaiterais plutôt embrasser la fin de ce chapitre et la conclusion pour complexifier le récit, à partir d'une vignette ethnographique qui est presque un conte (p. 195-197), illustrant de manière subtile le parallèle animal/humain, 
entre être « un animal mongol » (p. 183) et « un "vrai" Mongol » (p. 197), et ce dans un contexte où, comme mentionné plus haut, les éleveurs mongols sont autocritiques (n'hésitant pas à se décrire comme "paresseux » car nomadisant moins, etc., p. 202). Dans cette vignette, par une journée de fin d'automne à $-25^{\circ} \mathrm{C}$, deux éleveurs souhaitent abattre des bovins, en compagnie de leurs trois chiens, dont l'un seulement est de race mongole. Après avoir dépecé un premier bovin et laissé la viande découpée geler sur une charrette, les éleveurs décident de faire une pause thé pour se réchauffer, et attachent leurs chiens, à l'exception du mongol. Les deux autres ont contre eux leur race, mais aussi d'avoir été élevés à la ville. Cela vaut aussi pour les humain-es, puisqu' « un "vrai" Mongol est éleveur et vit à la campagne, érigeant le pastoralisme nomade en mode de vie mongol par excellence ». Au retour de la pause, la viande était intacte. Ce qui fait ici la force et la finesse du livre de Charlotte Marchina, c'est non seulement cette nouvelle manifestation d'une ambiance crépusculaire (puisqu'on peut aussi mettre en relation la critique de la russification des chiens et l'autocritique quant à la paresse de nomadiser, et qu'à chaque fois on laisse aux auxiliaires russes réaliser les activités censées représenter le «"vrai" Mongol»); mais aussi la restitution de la complexité des relations entre humains, animaux et environnement. L'auteure ne fait pas le récit d'une harmonie et d'une symbiose rêvées, mais celui, très concret et littéral de l'alternance voire de l'intermittence de ces relations, laissant émerger des formes d'autonomie et de détachement pour les uns ou les autres, et au final une grande flexibilité. C'est peut-être d'ailleurs précisément cet imaginaire symbiotique qui en est à son crépuscule, pour laisser place à des manières de nomadiser diverses, libérées de cet héritage douteux et impossible à mettre en œuvre.

\section{NOTES}

1. Donna Haraway, Manifeste des espèces compagnes : chiens, humains et autres partenaires, trad. de l'anglais par Jérôme Hansen, préf. de Vinciane Despret, Paris, Climats/Flammarion, 2019.

\section{AUTEURS}

\section{ANTHONY PECQUEUX}

Anthony.PECQUEUX[at]msh-lse.fr 